\title{
Effects of Cognitive Performance and Affective Status on Fatigue in Parkinson's Disease
}

\author{
Susan Spirgi ${ }^{a} \quad$ Antonia Meyer $^{a} \quad$ Pasquale Calabrese $^{b} \quad$ Ute Gschwandtner $^{a}$ \\ Peter Fuhr ${ }^{\text {a }}$ \\ ${ }^{a}$ Department of Neurology, University Hospital Basel, Basel, Switzerland; ${ }^{b}$ Department of \\ Psychology, University of Basel, Basel, Switzerland
}

Keywords

Parkinson's disease $\cdot$ Sleepiness · Fatigue $\cdot$ Cognition · Depression · Apathy

\begin{abstract}
Background: Fatigue is a common non-motor symptom in Parkinson's disease (PD) and is typically assessed via self-reported questionnaires such as the Parkinson's Fatigue Scale (PFS). The PFS captures the presence of subjective experience of physical fatigue as well as its impact on daily functioning. Objectives: We aimed to investigate whether different variables (cognition, neuropsychiatric symptoms, disease-related measures) are associated with the experience of physical fatigue in comparison to fatigue affecting daily functioning. Method: Sixtytwo non-demented PD patients were evaluated through questionnaires assessing fatigue, daytime sleepiness, apathy, depression, anxiety, and cognition. Items of fatigue were classified and summarized into two index variables measuring either the subjective experience of physical fatigue or the impact of fatigue on daily functioning. Linear regression with a stepwise elimination procedure was conducted to select the significant predictors for each index variable separately. Results: Subjective experience of physical fatigue (Model $1 ; r^{2}=0.46 ; p<0.01$ ) was significantly associated with higher levels of depression $(b=0.07 ; p<0.01)$, anxiety $(b=$ $0.03 ; p<0.05)$, and lower performances in verbal episodic memory $(b=-0.16 ; p<0.05)$. Fatigue affecting daily functioning (Model $2 ; r^{2}=0.44 ; p<0.05$ ) was significantly related to higher levels of depression ( $b=0.07 ; p<0.01)$, anxiety $(b=0.03 ; p>0.05)$, and lower motor functioning ( $b=0.01 ; p=0.05$ ). Conclusions: In conclusion, our work supports associations between fatigue and other neuropsychiatric symptoms in PD and extends prior work suggesting that motor disturbances are specifically linked to fatigue-related impairment of daily functioning, but not to the subjective experience of physical fatigue.




\section{Introduction}

Fatigue is a common non-motor symptom of Parkinson's disease (PD) with a prevalence of $50 \%$ [1]. It is considered as one of the most disabling symptoms, having a severe impact on daily living and the quality of life of affected individuals [2]. Fatigue refers to a subjective sense of exhaustion [3] and is typically assessed via self-reported questionnaires such as the Parkinson's Fatigue Scale (PFS). According to the two-concept model of Mosso [4], fatigue leads to a limitation of physical and cognitive function caused by an interaction between exhaustion and perception of fatigue. The PFS captures two effects of fatigue in PD [15] separately, i.e., the presence of the subjective experience of fatigue, whereby physical effects of fatigue are emphasized, and the impact of fatigue on daily functioning and activities, such as socialization and work. The pathophysiology of fatigue in PD is still unknown. A recent review [1] found no association between fatigue and motor signs, concluding that fatigue in PD might be caused by a disruption of non-dopaminergic pathways [1]; however, other studies clearly show a relationship between fatigue and motor impairments [11].

Besides fatigue, depression [5], anxiety [6] and apathy [7] are highly prevalent in PD. According to Siciliano et al., all these neuropsychiatric symptoms are associated with each other [1]. Other studies showed that fatigue can persist in spite of successfully treated depression, suggesting that the two syndromes are independent [8]. Additionally, fatigue was found to be influenced by excessive daytime sleepiness (EDS), which is described as undesirable and inappropriate sleepiness during waking hours [9].

Fatigue in non-demented PD patients is associated with cognitive impairment, particularly with executive dysfunction [12] and visuospatial deficits [13]. A study assessing the association between fatigue and the efficiency of the attention networks, found that the presence of fatigue is associated with a less efficient executive network [14].

The present study concurrently evaluates the relationship between cognition (verbal episodic memory, attention, executive function, and visuo-construction), motor signs, neuropsychiatric symptoms (apathy, depression, and anxiety) and self-rated fatigue in individuals with PD. Our aim was to investigate whether different variables are associated with physical fatigue in comparison to the impact of fatigue on daily functioning. A better understanding of the possible interaction between different aspects of fatigue could potentially characterize this disorder more precisely.

\section{Materials and Methods}

According to UK Parkinson's Disease Brain Bank Criteria [16] sixty-five patients with idiopathic PD were recruited for this study. Exclusion criteria were: dementia (MMSE score $\leq 24$ ), other severe brain disorders, alcohol or drug dependency, age $<45$ years and/or non idiopathic PD ( $n=2)$, insufficient knowledge of the German language, or more than 20\% missing data $(n=1)$. Consequently, the final data set comprised 62 ( 41 males and 21 females) subjects. All patients were on dopaminergic medication and were tested in the $\mathrm{ON}$ state.

\section{Psychiatric and Neuropsychological Assessment}

Fatigue was assessed using a German version of the PFS-16, a questionnaire with 16 items specifically developed for patients with PD [17]. The threshold for clinical significant fatigue is an average item score of $>2.95$. Seven items of the scale are related to the presence of a subjective experience of fatigue with physical effects (e.g., "I feel totally drained"), whereas nine items measure the effect of fatigue on daily functioning and activity [15] (e.g., "I get more tired than other people I know"). Accordingly, the PFS-16 items were assigned to either the 
first index variable, describing the subjective experience of physical fatigue, or the second, characterizing the effect of fatigue on daily functioning.

The following questionnaires were completed by the patients to assess symptoms of depression, apathy, anxiety and EDS:

Beck Depression Inventory-II (BDI-II) [18]

Apathy Evaluation Scale (AES-S) [19]

Beck Anxiety Inventory (BAI) [20]

Epworth Sleepiness Scale (ESS) [21]

As part of a comprehensive neuropsychological examination, the following cognitive domains were of interest for the present study: attention, executive function, verbal episodic memory, and visuo-construction. Z-scores of test variables (derived from raw scores with correction for age gender and education [22]) were averaged for each domain. The tests and their domain attribution were summarized as followed: Attention was measured by alertness (reaction time, with and without sound, of the computerized Test Battery of Attentional Performance [TAP] [23]) and the Trail Making Test, part A [24]. Executive function was measured by the Trail Making Test, part B, and verbal fluency (phonemic [25] and semantic fluency [26]). Verbal episodic memory was measured by the California Verbal Learning Test (CVLT) [27]. From the CVLT the following variables were extracted: Total trial 1-5, shortdelay free recall, long-delay free recall, and recognition-discriminability. Visuo-construction was measured by the Block Design Test [28] and the Rey-Osterrieth Complex Figure copy task [29].

\section{Neurological Assessment}

The severity of motor functioning was assessed by the Unified Parkinson's Disease Rating Scale (UPDRS) subscale III. The medication reported was calculated as the levodopa equivalent dosage [30].

\section{Statistical Analyses}

For statistical analyses IBM SPSS Statistics for Windows, version 24.0 [31], and R version 3.0.1 [32] were used. Correlation analyses were conducted with both indices of the PFS-16 and all variables of interest using Pearson's product-moment correlation coefficient or Spearman's rank correlation coefficients, depending on the significance of the Kolmogorov-Smirnov test.

In a next step, linear regression models with Akaike information criterion-based stepwise backwards elimination procedure were applied to select the relevant predictors of physical fatigue and of fatigue affecting daily functioning. Stepwise elimination procedures were performed using only significant correlations as potential predictors in accordance with the results of the correlation analyses.

$p$ values $<0.05$ were considered as statistically significant.

\section{Results}

Twenty-five of the examined patients had an item score higher than 2.95 in the PFS-16, indicating that $40 \%$ of the patients met the criteria of clinically relevant fatigue. Table 1 shows the properties of the sample.

In Table 2, the correlations between both indices of the PFS-16 and all variables of interest are shown. Both indices of the PFS-16 correlated positively with higher levels of the motor subscale in the UPDRS. Regarding the neuropsychiatric measures, higher values of physical fatigue were correlated with higher scores of apathy, depression, and anxiety (AES-S, BDI-II, 
Dementia

and Geriatric

Cognitive Disorders

Table 1. Sample description

Table 2. Correlation coefficients of the outcome variables of fatigue with all variables of interest

\begin{tabular}{l|l}
\hline Dement Geriatr Cogn Disord Extra 2019;9:344-351 \\
\hline DOI: 10.1159/000498883 & $\begin{array}{l}\text { (c) 2019 The Author(s). Published by S. Karger AG, Basel } \\
\text { www.karger.com/dee }\end{array}$ \\
\hline
\end{tabular}

Spirgi et al.: Effects of Cognitive Performance and Affective Status on Fatigue in PD

\begin{tabular}{lc}
\hline Characteristic & \multicolumn{1}{l}{ Value } \\
\hline Age, years & $67.5(34,72)$ \\
Education, years & $14.5(12,17)$ \\
MMSE & $29(28,30)$ \\
Disease duration, years & $8.5(4.25,14.75)$ \\
UPDRS (subscale III) & $10(6,19)$ \\
LED, mg/day & $577.25(325,792.25)$ \\
Fatigue_D & $2.70(2.02,3.55)$ \\
Fatigue_P & $2.21(1.75,2.85)$ \\
Apathy (AES-S) & $27(22.25,33.75)$ \\
Depression (BDI-II) & $6(4,10)$ \\
Anxiety (BAI) & $8(4.25,12.75)$ \\
Attention $^{\mathrm{a}}$ & $-0.71(-1.29,-0.14)$ \\
Executive function $^{\mathrm{a}}$ & $-0.30(-0.62,0.20)$ \\
Memory $^{\mathrm{a}}$ & $-1.20(-1.98,-0.13)$ \\
Visuoconstruction $^{\mathrm{a}}$ & $-0.16(-0.89,0.57)$ \\
\end{tabular}

The total number of patients was 62 (21 females). Values are presented as medians (quantiles). Quantiles refer to the 25th and 75 th percentiles. Fatigue_D, effect of fatigue on daily related functioning; Fatigue_P, physical effects of fatigue; MMSE, Mini Mental State Examination; LED, levodopa equivalent dosage. ${ }^{a}$ Averaged Z-scores with correction for age, gender, and education.

\begin{tabular}{lcc}
\hline & Fatigue_P & Fatigue_D \\
\hline Age & $0.02^{1}$ & $0.04^{1}$ \\
Education & $0.21^{2}$ & $0.19^{1}$ \\
MMSE & $-0.092^{1}$ & $-0.082^{1}$ \\
Disease duration, years & $-0.032^{1}$ & $-0.012^{1}$ \\
Daytime sleepiness (ESS) & $0.202^{1}$ & $0.172^{1}$ \\
UPDRS (subscale III) & $\mathbf{0 . 3 2}^{2}$ & $\mathbf{0 . 3 7}^{2}$ \\
LED (mg/day) & $0.15^{1}$ & $0.09^{1}$ \\
Apathy (AES-S) & $\mathbf{0 . 3 3}^{1}$ & $0.21^{1}$ \\
Depression (BDI-II) & $\mathbf{0 . 5 8}^{2}$ & $\mathbf{0 . 5 8}$ \\
Anxiety (BAI) & $\mathbf{0 . 5 5}^{2}$ & $\mathbf{0 . 5 4}$ \\
Attention & $-0.051^{1}$ & $-0.041^{1}$ \\
Executive function & $-0.042^{2}$ & $-0.021^{1}$ \\
Memory & $-\mathbf{0 . 2 6 1 ^ { 1 }}$ & $-0.241^{1}$ \\
Visuoconstruction & $-0.012^{2}$ & $0.03^{2}$ \\
\hline
\end{tabular}

Fatigue_P, subjective experience of physical fatigue; Fatigue_D, effect of fatigue on daily related functioning; LED, levodopa equivalent dosage; MMSE, Mini Mental State Examination.

$p$ values $<0.05$ are in bold.

Values are Spearman's ${ }^{1}$ rank or Pearson's product-moment ${ }^{2}$ correlation coefficients.

and BAI), whereas fatigue affecting daily functioning particularly correlated significantly with depression and anxiety, but not with apathy scores. For the cognitive domain, we observed only a significant relationship between physical fatigue and the verbal episodic memory performance (Table 2). In a further analysis, we observed that only the verbal episodic 
memory measure "recognition-discriminability" was correlated with higher levels of physical fatigue ( $r$ [62] $=-0.33 ; p<0.001$, one-tailed).

By stepwise elimination procedures the variables apathy and motor functioning (UPDRSIII) were excluded from the model explaining physical fatigue, whereas the parameters depression $(b=0.07 ; p<0.01)$, anxiety $(b=0.03 ; p<0.05)$, and verbal episodic memory performance (recognition-discriminability, $b=-0.16 ; p<0.05$ ) remained in the model. The overall model was significant $\left(r^{2}=0.49 ; p<0.001\right.$; adjusted $\left.r^{2}=0.46 ; F_{[3,58]}=18.88\right)$.

The model explaining the impact of fatigue on daily functioning was also significant $\left(r^{2}=\right.$ $0.44 ; p<0.01$; adjusted $\left.r^{2}=0.41 ; F_{[3,58]}=15.2\right)$ and was significantly associated with higher scores on BDI ( $b=0.07 ; p<0.01)$, BAI ( $b=0.03 ; p<0.05)$, and UPDRS-III ( $b=0.01 ; p=0.05)$.

\section{Discussion/Conclusion}

Subjective experience of physical fatigue is related to higher levels of depression, anxiety and lower verbal episodic memory performance, but not to more severe motor signs. The impact of fatigue on everyday activities shows similar associations with neuropsychiatric symptoms, but additionally predicts motor signs.

Although physical fatigue was significantly correlated with motor severity (UPDRS-III), the variable did not survive the stepwise regression procedure. In contrast, a higher impact of fatigue on daily functioning was significantly predicted by higher levels of motor impairment. We assume that motor impairments significantly reduce daily functioning, and therefore are a predictor of this aspect of fatigue. Consequently, this also may explain the lack of influence of motor function on physical fatigue, as it is not related to daily functioning. In accordance with the results of recent studies, we observed a strong association between levels of depression, anxiety, and fatigue $[1,34]$. It is argued that these symptoms considerably overlap with fatigue. For example the diagnosis of depression considers the presence of fatigue or a lack of energy as one of the core expressions of the disorder [35], and thus the symptoms of fatigue and depression describe may similar experiences [35]. This could potentially explain the significant correlation observed between fatigue and depression as they both have the same anatomical phenotype based on the classification system used by Sauerbier et al. [36]. Subtyping non-motor symptoms (NMS) in PD is a new concept. It is suggested that fatigueand depression-dominant NMS subtypes both belong to what is classified as a limbic phenotype [36].

We observed no significant correlation between fatigue and EDS, suggesting that symptoms of fatigue can occur independently from EDS. Fatigue is associated with exhaustion or a sensation of tiredness, either physical or mental [37] whereas sleepiness is described as a trigger signal for the onset of sleep [38]. The absence of a significant correlation between fatigue measured by the PFS-16 and EDS measured by the ESS (Table 2) is in line with previous findings $[11,39,40]$.

We observed a significant association between subjective experiences of physical fatigue and a lower performance on verbal episodic memory tasks, specifically with a reduced performance of recognition-discriminability (i.e., \% of correctly recognized words from CVLT-encoding). This is a novel finding and inconsistent with other studies indicating that fatigue is rather associated with visuospatial function in drug-naive PD patients [13] and executive dysfunctions. In the study of Kluger et al. [13] the domains of verbal episodic memory, processing speed, executive function, and visuospatial abilities were taken into account. They found a strong relationship between fatigue and visuospatial abilities, but not with verbal episodic memory. Accordingly, our finding needs to be replicated and further validated. 
So far, no official German translation or validation of the PFS-16 is available. The translation of the term fatigue used in the present study is translated by using a German word which also carries the meaning of tiredness. This is a strong limitation of the study and the PFS-16 score should therefore be interpreted as an approximation of the construct fatigue. In addition, self-rating questionnaires were used to assess neuropsychiatric symptoms; this requires both a specific level of motivation and cognitive functioning. Both aspects might be impaired in PD patients and thus reduce the informative value of such tools.

In conclusion, our work supports an influence of neuropsychiatric symptoms on fatigue in PD and extends prior work suggesting that motor disturbances are specifically linked to fatigue-related impairment of daily functioning, but not to the subjective experience of physical fatigue.

\section{Acknowledgement}

We thank the participating subjects and caregivers. We also thank the trainees for helping with the data acquisition and data entry. The financial support of the Swiss National Science Foundation (CR32I2_159682), the Hedwig Widmer-Stiftung, the Gossweiler Foundation, the Bangerter Foundation, the Freiwillige Akademische Gesellschaft Basel, Parkinson Schweiz, the Mach-Gaensslen-Stiftung, General Electrics, and AbbVie is gratefully acknowledged.

\section{Statement of Ethics}

This study was approved by the local ethics committee (Ethikkommission beider Basel, reference No. 135/11 and 294/13). All of the patients were fully informed of the nature of this study and written informed consent was given by all study participants.

\section{Disclosure Statement}

The authors have no conflict of interests to declare.

\section{Funding Sources}

Pasquale Calabrese has received honoraria for speaking at scientific meetings, serving on scientific advisory boards, and consulting activities from Abbvie, Actelion, Almirall, BayerSchering, Biogen, EISAI, Lundbeck, Merck Serono, Novartis, Sanofi-Aventis, and Teva. He has also received research grants from the Swiss Multiple Sclerosis Society (SMSG), and the Swiss National Research Foundation. Ute Gschwandtner received support for this research from the Mach-Gaensslen-Foundation, the Gossweiler Foundation, Parkinson Schweiz, the Synapsis Foundation, the Botnar Foundation, and the Hedwig-Widmer-Stiftung. Peter Fuhr received support for this research from the Swiss National Science Foundation, the Mach-Gaensslen Foundation, the Gossweiler Foundation, Parkinson Schweiz, the Synapsis Foundation, the Botnar Foundation, the Freiwillige Akademische Gesellschaft Basel, the Novartis Research Foundation, Novartis, Roche, AbbVie, and the Hedwig-Widmer-Stiftung.

All other authors declare that this research was conducted in the absence of any commercial or financial relationships that could be construed as a potential conflict of interest. 


\section{Author Contributions}

Susan Spirgi helped to conduct the study, performed data analyses, and drafted the this paper. Antonia Meyer supervised the neuropsychological examination, contributed to the analyses, and critically revised this paper. Ute Gschwandtner, Peter Fuhr, and Pasquale Calabrese conceived and designed this study, participated in its design and coordination, and helped to draft this paper and critically revised it. All of the authors read and approved the final version of this paper.

\section{References}

1 Siciliano M, Trojano L, Santangelo G, De Micco R, Tedeschi G, Tessitore A. Fatigue in Parkinson's disease: A systematic review and meta-analysis. Mov Disord. 2018 Nov;33(11):1712-23.

2 Dogan VB, Koksal A, Dirican A, Baybas S, Dirican A, Dogan GB. Independent effect of fatigue on health-related quality of life in patients with idiopathic Parkinson's disease. Neurol Sci. 2015 Dec;36(12):2221-6.

3 Friedman JH, Beck JC, Chou KL, Clark G, Fagundes CP, Goetz CG, et al. Fatigue in Parkinson's disease: report from a mutidisciplinary symposium. NPJ Parkinsons Dis. 2016;2(1):15025.

4 Enoka RM, Duchateau J. Translating Fatigue to Human Performance. Med Sci Sports Exerc. 2016 Nov;48(11): 2228-38.

5 Alzahrani H, Venneri A. Cognitive and neuroanatomical correlates of neuropsychiatric symptoms in Parkinson's disease: A systematic review. J Neurol Sci. 2015 Sep;356(1-2):32-44.

6 Broen MP, Narayen NE, Kuijf ML, Dissanayaka NN, Leentjens AF. Prevalence of anxiety in Parkinson's disease: A systematic review and meta-analysis. Mov Disord. 2016 Aug;31(8):1125-33.

7 Cubo E, Benito-León J, Coronell C, Armesto D; ANIMO Study Group. Clinical correlates of apathy in patients recently diagnosed with Parkinson's disease: the ANIMO study. Neuroepidemiology. 2012;38(1):48-55.

8 Alves G, Wentzel-Larsen T, Larsen JP. Is fatigue an independent and persistent symptom in patients with Parkinson disease? Neurology. 2004 Nov;63(10):1908-11.

9 Knie B, Mitra MT, Logishetty K, Chaudhuri KR. Excessive daytime sleepiness in patients with Parkinson's disease. CNS Drugs. 2011 Mar;25(3):203-12.

10 Valko PO, Waldvogel D, Weller M, Bassetti CL, Held U, Baumann CR. Fatigue and excessive daytime sleepiness in idiopathic Parkinson's disease differently correlate with motor symptoms, depression and dopaminergic treatment. Eur J Neurol. 2010 Dec;17(12):1428-36.

11 Havlikova E, van Dijk JP, Rosenberger J, Nagyova I, Middel B, Dubayova T, et al. Fatigue in Parkinson's disease is not related to excessive sleepiness or quality of sleep. J Neurol Sci. 2008 Jul;270(1-2):107-13.

12 Goldman JG, Stebbins GT, Leung V, Tilley BC, Goetz CG. Relationships among cognitive impairment, sleep, and fatigue in Parkinson's disease using the MDS-UPDRS. Parkinsonism Relat Disord. 2014 Nov;20(11):1135-9.

13 Kluger BM, Pedersen KF, Tysnes OB, Ongre SO, Øygarden B, Herlofson K. Is fatigue associated with cognitive dysfunction in early Parkinson's disease? Parkinsonism Relat Disord. 2017 Apr;37:87-91.

14 Pauletti C, Mannarelli D, Locuratolo N, Pollini L, Currà A, Marinelli L, et al. Attention in Parkinson's disease with fatigue: evidence from the attention network test. J Neural Transm (Vienna). 2017 Mar;124(3):335-45.

15 Friedman JH, Alves G, Hagell P, Marinus J, Marsh L, Martinez-Martin P, et al. Fatigue rating scales critique and recommendations by the Movement Disorders Society task force on rating scales for Parkinson's disease. Mov Disord. 2010 May;25(7):805-22.

16 Gibb WR, Lees AJ. The relevance of the Lewy body to the pathogenesis of idiopathic Parkinson's disease. J Neurol Neurosurg Psychiatry. 1988 Jun;51(6):745-52.

17 Brown RG, Dittner A, Findley L, Wessely SC. The Parkinson fatigue scale. Parkinsonism Relat Disord. 2005 Jan; 11(1):49-55.

18 Hautzinger M, Bailer M, Worall H, Keller F. BDI Beck-Depressions-Inventar Testhandbuch. 2. Bern: HansHuber Verlag; 1995.

19 Lueken U, Seidl U, Schwarz M, Völker L, Naumann D, Mattes K, et al. [Psychometric properties of a German version of the Apathy Evaluation Scale]. Fortschr Neurol Psychiatr. 2006 Dec;74(12):714-22.

20 Ehlers A, Margraf J: Deutschsprachige Adaptation des Beck Anxiety Inventory von A. T. Beck und R. A. SternHogrefe Verlagsgruppe, 2007.

21 Johns MW. A new method for measuring daytime sleepiness: the Epworth sleepiness scale. Sleep. 1991 Dec; 14(6):540-5.

22 Berres M, Zehnder A, Bläsi S, Monsch AU. Evaluation of diagnostic scores with adjustment for covariates. Stat Med. 2008 May;27(10):1777-90.

23 Zimmermann P, Fimm B. Testbatterie zur Aufmerksamkeitsprüfung. Herzogenrath: Psytest; 2007.

24 Reitan RM. The relation of the trail making test to organic brain damage. J Consult Psychol. 1955 Oct;19(5): 393-4. 
25 Thurstone LL. Primary mental abilities. Science. 1948 Nov;108(2813):585.

26 Isaacs B, Kennie AT. The Set test as an aid to the detection of dementia in old people. Br J Psychiatry. 1973 Oct; 123(575):467-70.

27 Delis D, Kramer J, Ober B, Kaplan E. The California Verbal Learning Test: Administration and interpretation San Antonio. TX: Psychological Corporation; 1987.

28 Härtig C, Markowitsch HJ, Neufeld H, Calabrese P, Deisinger K, Kessler J. Wechsler Gedächtnis Test - Revidierte Fassung. Bern: Verlag Hans Huber; 2000.

29 Duley JF, Wilkins JW, Hamby SL, Hopkins DG, Burwell RD, Barry NS. Explicit scoring criteria for the Rey-Osterrieth and Taylor complex figures. Clin Neuropsychol. 1993;7(1):29-38.

30 Tomlinson CL, Stowe R, Patel S, Rick C, Gray R, Clarke CE. Systematic review of levodopa dose equivalency reporting in Parkinson's disease. Mov Disord. 2010 Nov;25(15):2649-53.

31 IBM Corporation: IBM SPSS Statistics for Windows. Version 24.0 [software]. Armonk: IBM; 2016.

32 R Core Team. R: a language and environment for statistical computing [software]. Available from: http:// www.R-project.org/.

33 Svensson E. Fatigue and sleepiness in Parkinson's disease patients: disorders of Sleep and circadian rhythms in Parkinson's disease. Vienna: Springer; 2015. p. 173-81.

34 Friedman JH, Brown RG, Comella C, Garber CE, Krupp LB, Lou JS, et al.; Working Group on Fatigue in Parkinson's Disease. Fatigue in Parkinson's disease: a review. Mov Disord. 2007 Feb;22(3):297-308.

35 World Health Organization. ICD-10: international statistical classification of diseases and related health problems. Geneva: WHO; 2016.

36 Sauerbier A, Jenner P, Todorova A, Chaudhuri KR. Non motor subtypes and Parkinson's disease. Parkinsonism Relat Disord. 2016 Jan;22 Suppl 1:S41-6.

37 Fu R, Luo XG, Ren Y, He ZY, Lv H. Clinical characteristics of fatigued Parkinson's patients and the response to dopaminergic treatment. Transl Neurodegener. 2016 May;5(1):9.

38 Neu D, Mairesse O, Hoffmann G, Valsamis JB, Verbanck P, Linkowski P, et al. Do 'sleepy' and 'tired' go together? Rasch analysis of the relationships between sleepiness, fatigue and nonrestorative sleep complaints in a nonclinical population sample. Neuroepidemiology. 2010;35(1):1-11.

39 Herlofson K, Ongre SO, Enger LK, Tysnes OB, Larsen JP. Fatigue in early Parkinson's disease. Minor inconvenience or major distress? Eur J Neurol. 2012 Jul;19(7):963-8.

40 Wen MC, Chan LL, Tan LC, Tan EK. Depression, anxiety, and apathy in Parkinson's disease: insights from neuroimaging studies. Eur J Neurol. 2016 Jun;23(6):1001-19. 